\title{
Application of Gravity Gradients in the Process of GOCE Orbit Determination
}

\author{
Andrzej BOBOJĆ \\ University of Warmia and Mazury in Olsztyn, \\ Department of Astronomy and Geodynamics, Olsztyn, Poland \\ e-mail: andrzej.bobojc@gmail.com
}

\begin{abstract}
The possibility of improving the Gravity Field and Steady-State

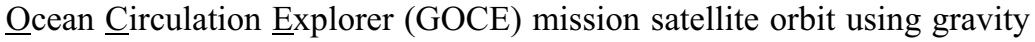
gradient observations was investigated. The orbit improvement is performed by a dedicated software package, called the Orbital Computation System (OCS), which is based on the classical least squares method. The corrections to the initial satellite state vector components are estimated in an iterative process, using dynamic models describing gravitational perturbations. An important component implemented in the OCS package is the 8 th order Cowell numerical integration procedure, which directly generates the satellite orbit. Taking into account the real and simulated GOCE gravity gradients, different variants of the solution of the orbit improvement process were obtained. The improved orbits were compared to the GOCE reference orbits (Precise Science Orbits for the GOCE satellite provided by the European Space Agency) using the root mean squares (RMS) of the differences between the satellite positions in these orbits. The comparison between the improved orbits and the reference orbits was performed with respect to the inertial reference frame (IRF) at J2000.0 epoch. The RMS values for the solutions based on the real gravity gradient measurements are at a level of hundreds of kilometers and more. This means that orbit improvement using the real gravity gradients is ineffective. However, all solutions using simulated gravity gradients have RMS values below the threshold determined by the RMS
\end{abstract}

Ownership: Institute of Geophysics, Polish Academy of Sciences;

(C) 2016 Bobojć. This is an open access article distributed under the Creative Commons Attribution-NonCommercial-NoDerivs license,

http://creativecommons.org/licenses/by-nc-nd/3.0/. 
values for the computed orbits (without the improvement). The most promising results were achieved when short orbital arcs with lengths up to tens of minutes were improved. For these short arcs, the RMS values reach the level of centimeters, which is close to the accuracy of the Precise Science Orbit for the GOCE satellite. Additional research has provided requirements for efficient orbit improvement in terms of the accuracy and spectral content of the measured gravity gradients.

Key words: GOCE satellite orbit, gravity gradients.

\section{INTRODUCTION}

The Gravity Field and Steady-State Ocean Circulation Explorer (GOCE) mission terminated in November 2013 was dedicated to the study of the Earth's gravitational field. For the first time, a satellite with a gradiometer on board was successfully placed in an almost circular orbit with an inclination of $96.7^{\circ}$ and a mean altitude of around $254.9 \mathrm{~km}$ (Rummel et al. 2009). The gradiometer, consisting of three pairs of identical accelerometers, forming three arms, each around $50 \mathrm{~cm}$ long, provided the measurements of the second spatial derivatives of the geopotential, also known as gravity gradients, or gravity gradient tensor (GGT) measurements. The second instrument, besides the gradiometer installed on board the GOCE satellite, was a Global Positioning System (GPS) receiver, which was the source of code and phase observations of the GPS satellite constellation, called Satellite-to-Satellite Tracking high-low mode observations (SST-hl) or simply GPS observations (ESA 1999). Both mentioned types of measurements, collected during the four years of the GOCE mission, are the basic data serving to implement the main scientific aim, i.e., the estimation of new models of the Earth's static gravity field. These models in the form of sets of coefficients of geopotential expansion into a spherical harmonic series will find application in a number of sciences such as solid Earth physics, oceanography, and geodesy (Johannessen et al. 2003). An important factor in the determination of these models is the geolocation of observation data, performed by satellite orbit estimation. This estimation is carried out using SST-hl measurements, which are composed of the above-mentioned dual-frequency code and phase observations with respect to GPS satellites.

The determined GOCE satellite orbit occurs in two versions. The first version is based on parameters describing the field of forces determining the satellite's motion, including, first of all, the gravity field model. Orbit determination starts with the preparation of data on GPS satellites: orbital information and their clock corrections. Parameters describing Earth rotation are also taken into account (Bock et al. 2007, 2011). The approximate orbit is generated in the first stage using pseudo-range measurements. This orbit is 
described by six osculating Keplerian elements at the initial epoch and nine empirical parameters modeling the constant and periodically changing additional satellite acceleration. The approximate orbit serves to synchronize the clock of the GPS receiver onboard the satellite with the GPS time system for all observation epochs. In the next step, the aforementioned orbit is improved by means of an iterative procedure using phase measurements with a 30 -s sampling interval. This time, the obtained orbit has a richer parameterization; the above-mentioned six osculating Keplerian elements at the initial epoch and nine empirical parameters are additionally supplemented by three components of the pseudo-stochastic acceleration vector, estimated for successive 15-min intervals (Bock et al. 2007). The next stage is another orbit improvement using phase observations with an even higher time resolution than before, which is now at $10 \mathrm{~s}$. The final orbit, obtained after an appropriate number of iterations, is defined by the six osculating elements at the initial epoch, three empirical constants of satellite acceleration in the radial direction, along the direction of motion and in the direction perpendicular to the instantaneous plane of the orbit, respectively, and satellite accelerations constant at successive 6-min intervals along the directions listed above. The GPS measurements mentioned here are non-differentiated observations of the carrier phase, hence the need for additional determination of the ambiguities of the total number of cycles and the receiver clock corrections. The obtained satellite orbit is presented as a time series of position and velocity components at 10-s intervals relative to the International Terrestrial Reference Frame 2005 (ITRF2005) reference frame (ESA 2010). The first version of the GOCE orbit described above is referred to as the reduced-dynamic orbit because it partly depends on the estimated pseudo-stochastic vectors of the satellite's empirical acceleration. For the second version of the GOCE orbit, also called the kinematic orbit, the determination is based solely on geometric data (code and phase measurements); no parameters characterizing the dynamic state of the satellite are taken into account. As a result, only the satellite position vectors for successive epochs are determined together with the ambiguity of the total number of cycles and the receiver clock corrections. The final solution is a time series of the satellite's coordinates in the ITRF2005 reference frame with 1-s time resolution (Bock et al. 2007).

To assess external accuracy, both versions of the GOCE orbit were compared with the orbit determined based on the Satellite Laser Ranging (SLR) measurements. The obtained differences show the accuracy of determining the reduced-dynamic orbit and the kinematic orbit at a level of a few centimeters (Bock et al. 2011).

As shown by the above description, the process of determining both versions of the GOCE satellite orbit is based on the use of GPS observations, while the second type of measurements from the GOCE mission (gravity 
gradients) finds application in the estimation of gravity field models. However, gravity gradients as the second spatial derivatives of the geopotential also contain information about the satellite's position. The possibility of using this information was noted by Rummel and Colombo (1985), where the corrections to the spherical harmonic coefficients were determined together with the corrections to the initial satellite state vector components. Bobojć and Drożyner (2003) showed the possibility of using the gravity gradient tensor components to improve simulated low Earth-orbiting (LEO) satellite orbits. An interesting idea of the application of dynamical aspect of gravity gradients for a satellite orbit determination is given by Eshagh (2014). The satellite acceleration vector is derived from gravity gradients in order to determine an orbit by the numerical integration (Eshagh 2014).

The aim of this paper is to examine the possibility and conditions of efficient GOCE satellite orbit determination using the geometrical information contained in gravity gradient observations.

\section{THEORETICAL BASIS}

The satellite's motion in the Earth's gravity field can be described by an equation in the following general form (Palacios et al. 1992):

$$
\ddot{r}=-\frac{\mathrm{GM}}{r^{3}} r+f,
$$

where $\boldsymbol{r}$ and $\ddot{\boldsymbol{r}}$ are the satellite position and acceleration vectors, respectively, $r$ is the distance between the satellite and the Earth's mass center, GM is the product of the gravity constant and Earth's mass, $\boldsymbol{f}$ is the perturbing acceleration vector. The first term of the right-hand side of Eq. 1 represents the satellite's Keplerian motion in the central gravity field, the other takes into account the effect of perturbing forces, which can be divided into two basic groups. The first of them includes gravitational perturbations generated by the geopotential, Earth tides, ocean tides, the Earth's rotational deformation caused by changes in the angular velocity vector, the third body effect and the relativity effects. The other group contains non-gravitational perturbations: atmospheric drag, solar radiation pressure, the albedo effect, and the effect of magnetic forces (Eshagh and Najafi-Alamdari 2007). The resultant perturbing acceleration vector $\boldsymbol{f}$ can also be composed (besides accelerations resulting from the above-mentioned forces) of pseudo-stochastic empirical acceleration vectors constant at specific time intervals, which were estimated during the determination of the reduced-dynamic orbit in the case of the GOCE satellite (Bock et al. 2011).

It is obvious that the dominant effect on the satellite's motion is exerted by the gravity field, whose potential $V$ is expressed by (Heiskanen and Moritz 1967): 


$$
\begin{aligned}
V(r, \theta, \lambda) & =W(r, \theta, \lambda)-Z(r, \theta, \lambda) \\
& =\frac{\mathrm{GM}}{r} \sum_{n=0}^{N_{\max }}\left(\frac{a}{r}\right)^{n} \sum_{m=0}^{n}\left(\bar{C}_{n m} \cos m \lambda+\bar{S}_{n m} \sin m \lambda\right) \bar{P}_{n m}(\cos \theta) .
\end{aligned}
$$

In Eq. $2 r, \theta$, and $\lambda$ are the geocentric coordinates of a given point; $r$ is the distance from Earth's center, $\theta=90^{\circ}-\varphi$ is the colatitude and $\varphi$ the geocentric latitude, $\lambda$ is the geocentric longitude, $W(r, \theta, \lambda)$ is the gravity potential containing centrifugal potential, $Z(r, \theta, \lambda)$ is the centrifugal potential, $a$ is the equatorial radius of the Earth ellipsoid, $\bar{C}_{n m}, \bar{S}_{n m}$ are the spherical harmonic coefficients (Stokes' coefficients) of degree $n\left(n=0,1, \ldots, N_{\max } ; N_{\max }\right.$ is maximum degree of the spherical harmonic expansion) and order $m$ ( $m=$ $0,1, \ldots, n)$, and $\bar{P}_{n m}(\cos \theta)$ is the normalized associated Legendre function of degree $n$ and order $m$.

As already noted, the gravity gradients $V_{i j}(i, j=1,2,3)$ provided by the GOCE mission are the second spatial derivatives of the geopotential $V$. Hence, one can write

$$
V_{i j}=\frac{\partial^{2} V(r, \theta, \lambda)}{\partial x_{j} \partial x_{i}},
$$

where the geocentric coordinates $r, \theta, \lambda$ are functions of the Cartesian coordinates $x_{i}, x_{j}\left(i, j=1,2,3\right.$ and $\left.x_{1}=x, x_{2}=y, x_{3}=z\right)$ in the adopted Earth fixed reference frame. The gravity gradients form a second-order tensor field. This tensor, also called the gravity gradient tensor (GGT), or the Marussi tensor, assumes the form (Rummel et al. 2011)

$$
\boldsymbol{M}=\left[\begin{array}{ccc}
V_{x x} & V_{x y} & V_{x z} \\
V_{y x} & V_{y y} & V_{y z} \\
V_{z x} & V_{z y} & V_{z z}
\end{array}\right],
$$

where, for example, $V_{x y}=\partial^{2} V / \partial y \partial x$. The matrix $\boldsymbol{M}$ is symmetric and traceless, satisfying in the space external to the Earth the Laplace equation

$$
V_{x x}+V_{y y}+V_{z z}=0
$$

Taking into account the form of Eq. 2, which describes the geopotential, dependence on the coordinates $x, y, z$ can be found also for the gravity gradients $V_{i j}$, forming the tensor $\boldsymbol{M}$. Therefore, $V_{i j}$ measurements performed by the gradiometer onboard the satellite contain usable orbital information. 


\section{GOCE SATELLITE ORBIT IMPROVEMENT}

Assuming as the data the gravity gradient tensor components, the satellite orbit determination process can be based on the following observation equation:

$$
V_{i j k}^{o}+v_{i j k}=V_{i j k}^{c}+\frac{\partial V_{i j k}^{c}}{\partial \boldsymbol{r}} \frac{\partial \boldsymbol{r}}{\partial\left(\boldsymbol{r}_{o}, \dot{\boldsymbol{r}}_{o}\right)}\left[\begin{array}{c}
\Delta \boldsymbol{r}_{o} \\
\Delta \dot{\boldsymbol{r}}_{o}
\end{array}\right] .
$$

In Eq. 6:

$$
V_{i j k}^{o}, V_{i j k}^{c}-\text { observed and computed gravity gradient at epoch } k \text {, }
$$

$$
(i, j=x, y, z) \text {, respectively, }
$$

$v_{i j k}$ - correction to the observed $q$ of $V_{i j k}^{o}$,

$\frac{\partial V_{i j k}^{c}}{\partial \boldsymbol{r}}$ - partial derivative of the computed gravity gradient $V_{i j k}^{c}$ with respect

to the satellite position vector $\boldsymbol{r}=[x, y, z]^{\mathrm{T}}$ at epoch $k$,

$\boldsymbol{r}_{o}, \dot{\boldsymbol{r}}_{o}$ - initial satellite position vector and initial satellite velocity vector,

respectively, at initial epoch $t_{o} ; \boldsymbol{r}_{o}=\left[x_{o}, y_{o}, z_{o}\right]^{\mathrm{T}}$ and $\dot{\boldsymbol{r}}_{o}=\left[\dot{x}_{o}, \dot{y}_{o}, \dot{z}_{o}\right]^{\mathrm{T}}$, $\frac{\partial \boldsymbol{r}}{\partial\left(\boldsymbol{r}_{o}, \dot{\boldsymbol{r}}_{o}\right)}-$ partial derivative of the position vector with respect to the initial state vector $\boldsymbol{p}_{o}=\left[\boldsymbol{r}_{o}, \dot{\boldsymbol{r}}_{o}\right]^{\mathrm{T}}$ interpolated at epoch $k$, $\Delta \boldsymbol{r}_{o}, \Delta \dot{\boldsymbol{r}}_{o}$ - vectors of corrections to the initial satellite position vector and the initial satellite velocity vector; $\Delta \boldsymbol{r}_{o}=\left[\Delta x_{o}, \Delta y_{o}, \Delta z_{o}\right]^{\mathrm{T}}$ and $\Delta \boldsymbol{r}_{o}=\left[\Delta \dot{x}_{o}, \Delta \dot{y}_{o}, \Delta \dot{z}_{o}\right]^{\mathrm{T}}$.

The gravity gradient $V_{i j k}^{c}$ at epoch $k$ from Eq. 6 is determined as the second-order partial derivative of geopotential (Eq. 2) with respect to the rectangular coordinates $x, y$, and $z$ of the satellite in the Earth-fixed reference frame. On the other hand, the quantity $\partial V_{i j k}^{c} / \partial \boldsymbol{r}$ at epoch $k$, consisting of the components $\partial V_{i j k}^{c} / \partial x, \partial V_{i j k}^{c} / \partial y, \partial V_{i j k}^{c} / \partial z$, is computed as the third-order spatial derivatives of the geopotential, taking into account the satellite position in the Earth-fixed reference frame. These derivatives form the thirdorder tensor. The detailed formulas used for the computation of $V_{i j k}^{c}$ and $\partial V_{i j k}^{c} / \partial \boldsymbol{r}$ are taken from Metris et al. (1999), where the problem of computing the spatial derivatives of the geopotential of any order was solved. The partial derivative of the position vector with respect to the initial state vector, occurring also in Eq. 6, forms the following matrix: 


$$
\frac{\partial \boldsymbol{r}}{\partial\left(\boldsymbol{r}_{o}, \dot{\boldsymbol{r}}_{o}\right)}=\left[\begin{array}{cccccc}
\frac{\partial x}{\partial x_{o}} & \frac{\partial x}{\partial y_{o}} & \frac{\partial x}{\partial z_{o}} & \frac{\partial x}{\partial \dot{x}_{o}} & \frac{\partial x}{\partial \dot{y}_{o}} & \frac{\partial x}{\partial \dot{z}_{o}} \\
\frac{\partial y}{\partial x_{o}} & \frac{\partial y}{\partial y_{o}} & \frac{\partial y}{\partial z_{o}} & \frac{\partial y}{\partial \dot{x}_{o}} & \frac{\partial y}{\partial \dot{y}_{o}} & \frac{\partial y}{\partial \dot{z}_{o}} \\
\frac{\partial z}{\partial x_{o}} & \frac{\partial z}{\partial y_{o}} & \frac{\partial z}{\partial z_{o}} & \frac{\partial z}{\partial \dot{x}_{o}} & \frac{\partial z}{\partial \dot{y}_{o}} & \frac{\partial z}{\partial \dot{z}_{o}}
\end{array}\right]
$$

whose elements are propagated in the numerical integration of GOCE orbit using the 8th order Cowell method, starting from the initial values $\partial_{i} / \partial_{j}=\delta_{i j}$ ( $i=x, y, z ; j=x_{o}, y_{o}, z_{o} ; \delta_{i j}$ - the Kronecker delta).

The application of the classical least squares method with an iterative approach leads to the determination of the vector $\Delta \boldsymbol{p}_{\boldsymbol{o} i}$ of unknown corrections to the initial satellite state vector for the $i$ th iteration using the following formula:

$$
\Delta \boldsymbol{p}_{o_{i}}=\left[\Delta \boldsymbol{r}_{o_{i}}, \Delta \dot{\boldsymbol{r}}_{o_{i}}\right]^{\mathrm{T}}=\left(\mathbf{A}_{i}^{\mathrm{T}} \mathbf{A}_{i}\right)^{-1} \mathbf{A}_{i}^{\mathrm{T}} \boldsymbol{l}_{i}
$$

in which the matrix $\mathbf{A}_{i}$ contains coefficients of observation equations equal to the partial derivatives of the gravity gradients with respect to the initial state vector, and the components of the vector $\boldsymbol{l}_{i}$ are differences between the observed and computed quantities ("O"-“C"). Thus, the matrix $\mathbf{A}_{i}$ and the vector $\boldsymbol{l}_{i}$ can be presented as:

$$
\begin{gathered}
A_{i}=\left[\mathbf{A}_{1} \mathbf{A}_{2} \cdots \mathbf{A}_{n}\right]^{\mathbf{T}}, \\
\boldsymbol{l}_{i}=\left[\boldsymbol{l}_{1} \boldsymbol{l}_{2} \cdots \boldsymbol{l}_{n}\right]^{\mathbf{T}},
\end{gathered}
$$

where submatrices $\mathbf{A}_{1}, \mathbf{A}_{2}, \ldots, \mathbf{A}_{n}$, and subvectors $\boldsymbol{l}_{1}, \boldsymbol{l}_{2}, \ldots, \boldsymbol{l}_{n}$ correspond to the successive observation epochs, and $n$ is the total numer of observation epochs. The submatrix $\mathbf{A}_{k}$ and subvector $\boldsymbol{l}_{k}$ at the given observation epoch $k(k=1,2, \ldots, n)$ are expressed by:

$$
\mathbf{A}_{k}=\left[\begin{array}{cc}
\frac{\partial V_{x x}^{c}}{\partial \boldsymbol{r}} \cdot \frac{\partial \boldsymbol{r}}{\partial\left(\boldsymbol{r}_{o}, \dot{\boldsymbol{r}}_{o}\right)} \\
\frac{\partial V_{y y}^{c}}{\partial \boldsymbol{r}} \cdot \frac{\partial \boldsymbol{r}}{\partial\left(\boldsymbol{r}_{o}, \dot{\boldsymbol{r}}_{o}\right)} \\
\frac{\partial V_{z z}^{c}}{\partial \boldsymbol{r}} \cdot \frac{\partial \boldsymbol{r}}{\partial\left(\boldsymbol{r}_{o}, \dot{\boldsymbol{r}}_{o}\right)} \\
\frac{\partial V_{x y}^{c}}{\partial \boldsymbol{r}} \cdot \frac{\partial \boldsymbol{r}}{\partial\left(\boldsymbol{r}_{o}, \dot{\boldsymbol{r}}_{o}\right)} \\
\frac{\partial V_{x z}^{c}}{\partial \boldsymbol{r}} \cdot \frac{\partial \boldsymbol{r}}{\partial\left(\boldsymbol{r}_{o}, \dot{\boldsymbol{r}}_{o}\right)} \\
\frac{\partial V_{y z}^{c}}{\partial \boldsymbol{r}} \cdot \frac{\partial \boldsymbol{r}}{\partial\left(\boldsymbol{r}_{o}, \dot{\boldsymbol{r}}_{o}\right)}
\end{array}\right], \quad l_{k}=\left[\begin{array}{c}
V_{x x}^{o}-V_{x x}^{c} \\
V_{y y}^{o}-V_{y y}^{c} \\
V_{z z}^{o}-V_{z z}^{c} \\
V_{x y}^{o}-V_{x y}^{c} \\
V_{x z}^{o}-V_{x z}^{c} \\
V_{y z}^{o}-V_{y z}^{c}
\end{array}\right] .
$$


For example:

$$
\frac{\partial V_{x x}^{c}}{\partial \boldsymbol{r}} \frac{\partial \boldsymbol{r}}{\partial\left(\boldsymbol{r}_{o}, \dot{\boldsymbol{r}}_{o}\right)}=\left[\frac{\partial V_{x x}^{c}}{\partial x_{o}} \frac{\partial V_{x x}^{c}}{\partial y_{o}} \frac{\partial V_{x x}^{c}}{\partial z_{o}} \frac{\partial V_{x x}^{c}}{\partial \dot{x}_{o}} \frac{\partial V_{x x}^{c}}{\partial \dot{y}_{o}} \frac{\partial V_{x x}^{c}}{\partial \dot{z}_{o}}\right] .
$$

Subsequent elements of the above expression can be presented analogously to the first element development, i.e.

$$
\frac{\partial V_{x x}^{c}}{\partial x_{o}}=\frac{\partial V_{x x}^{c}}{\partial x} \frac{\partial x}{\partial x_{o}}+\frac{\partial V_{x x}^{c}}{\partial y} \frac{\partial y}{\partial x_{o}}+\frac{\partial V_{x x}^{c}}{\partial z} \frac{\partial z}{\partial x_{o}} .
$$

In order to realize the aim of this work, the satellite orbit is improved by dedicated software called Orbital Computation System (OCS). It was created as an expansion of the Torun Orbit Processor (TOP) package (Drożyner 1995), which determines an orbit in the field of gravitational and nongravitational forces. In the OCS package, as already mentioned, the corrections to the initial dynamic state vector are estimated in successive iterations using the gravity gradient observations by means of the least squares method (Eq. 8). This means that the initial state vector $\boldsymbol{p}_{o_{i}-1}$ determined in the previous iteration is corrected within the current iteration to its actual value $\boldsymbol{p}_{o_{i}}$, which can be written as

$$
\boldsymbol{p}_{o_{i}}=\boldsymbol{p}_{o_{i-1}}+\Delta \boldsymbol{p}_{o_{i}},
$$

where $\Delta \boldsymbol{p}_{o_{i}}$ denotes the correction vector obtained in the current iteration. This iterative process ends after meeting the convergence criteria, taking into account the RMS difference between the observed gravity gradient $V_{i j k}^{o}$ and the computed gravity gradient $V_{i j k}^{c}$ for the previous iteration $i-1$ and the current iteration $i$. The convergence criteria are satisfied if

$$
\left|\frac{\mathrm{RMS}_{i}-\mathrm{RMS}_{i-1}}{\mathrm{RMS}_{i}}\right|<10^{-2} .
$$

\subsection{Algorithm for orbit determination}

In the frame of the OCS package, the orbit improvement process in a given iteration $i$ consists of the following three basic parts:

(1) The first part includes reading initial data, i.e., the initial conditions initial satellite state vector and options characterizing a given adjustment variant, the time series of gravity gradient observations, dynamic model parameters - coefficients of geopotential expansion into a spherical harmonic 
series, tidal model coefficients, third body position vectors, optionally the parameters of the empirical acceleration model, the time series of the elements of orientation of the ITRF2005 and the GOCE satellite's gradiometer reference frame (GRF) (ESA 2008) relative to the Cartesian inertial reference frame (IRF), which is realized based on the mean equator and vernal equinox of the J2000.0 standard epoch (ESA 2008). The time series of gravity gradient measurements and the above-mentioned orientation elements were acquired from the GOCE mission through the European Space Agency (ESA).

(2) The second part leads to determine the normal matrix $\left(\mathbf{A}_{i}^{\mathrm{T}} \mathbf{A}_{i}\right)^{-1}$ and the vector $\mathbf{A}_{i}^{\mathrm{T}} \boldsymbol{l}_{i}$ (Eq. 8). It is a process that takes place in a closed loop containing a number of repetitions equal to the number of observation epochs. The given repetition starts with the determination of transformation matrices at the given observation epoch between the ITRF2005 and GRF reference frames and between the ITRF2005 and IRF reference frames. This determination uses the aforementioned orientation elements which are given in terms of quarternions (ESA 2010).

Next, the computation of the satellite state vector (position and velocity vector) for the measurement epoch using numerical integration of equations of motion by the 8th order Cowell method and position interpolation for the measurement epoch is performed. A mathematical model of the forces acting on the satellite is created for the given epoch during the computations (Drożyner 1995). It takes into account gravitational perturbing forces and additionally optionally empirical accelerations. This model includes accelerations generated by the geopotential, ocean tides, Earth tides, the third body effect, and the relativity effects. Non-gravitational forces were compensated by the Drag-Free and Attitude Control System (DFACS) installed onboard the GOCE satellite, in which ion propulsion played an important role (Rebhan et al. 2000). The geopotential is represented by the ITG-GRACE2010S model (Mayer-Gürr et al. 2010). In this place, the derivatives of the vector of position with respect to the components of the initial state vector are also numerically propagated and interpolated for the observation epoch.

In the following step of the described part, the a priori values of measurements of gravity gradients (the second-order tensor) and the components of the third-order tensor of spatial derivatives $\partial V_{i j k}^{c} / \partial \boldsymbol{r}$ are computed in the ITRF2005 Earth-fixed reference frame, taking into account the determined position vector. This vector was transformed into the ITRF2005 frame from the IRF frame by means of the corresponding transformation matrix. Then, the observed tensor of gravity gradients, the computed (a priori) tensor of 
gravity gradients and the third-order tensor are transformed into the IRF reference frame, where the orbit improvement is performed.

The next step includes the calculation of residuals, i.e., the differences between the observed and a priori values of gravity gradients at the given measurement epoch. All of the above calculations allow to determine the partial values of the normal matrix $\left(\mathbf{A}_{i}^{\mathrm{T}} \mathbf{A}_{i}\right)^{-1}$ and the vector $\mathbf{A}_{i}^{\mathrm{T}} \boldsymbol{l}_{i}$. These values are summed in the successive repetitions. After the last repetition, the full normal matrix $\left(\mathbf{A}_{i}^{\mathrm{T}} \mathbf{A}_{i}\right)^{-1}$ and the full vector $\mathbf{A}_{i}^{\mathrm{T}} \boldsymbol{l}_{i}$ are obtained.

(3) The third part includes the determination of correction vector to the initial satellite state vector $\Delta \boldsymbol{p}_{o_{i}}=\left[\Delta x_{0}, \Delta y_{0}, \Delta z_{0}, \Delta \dot{x}_{0}, \Delta \dot{y}_{0}, \Delta \dot{z}_{0}\right]^{\mathrm{T}}$. This makes it possible to improve the initial state vector in the iteration $i$ using Eq. 14. In order to assess an internal accuracy of the orbit improvement process, RMS difference between the observed and computed (a priori) values of gravity gradient, the observation error of unit weight and standard deviations of corrected components of the vector $\boldsymbol{p}_{o_{i}}$ are estimated.

It should be noted that the a priori gravity gradients determined in the next iteration $i+1$ are computed along the satellite orbit improved in the previous iteration $i$.

\subsection{Parameter for the estimation of orbit determination accuracy}

For all orbital arcs, the initial state vectors before starting the orbit improvement process were equal to the initial state vectors of the corresponding reference orbit arcs. The corrected initial satellite state vector $\boldsymbol{p}_{o}$ allows to obtain an finally improved satellite orbit, through the previously-mentioned numerical integration by the 8th order Cowell method using the dynamic models listed above. The improved GOCE orbit is then compared with the reference orbit, to determine the key parameter for the assessment of the quality of the obtained solution. This parameter, denoted as $\mathrm{RMS}_{\mathrm{OUT}}$, characterizes the accuracy of the determined orbit. It is computed using the formula

$$
\mathrm{RMS}_{\text {OUT }}=\sqrt{\sum_{i=1}^{3}\left(\mathrm{RMS}_{i}\right)^{2}},
$$

with the quantities of $\operatorname{RMS}_{i}(i=1,2,3)$ obtained using the following expression:

$$
\mathrm{RMS}_{i}=\sqrt{\frac{\sum_{j=1}^{n}\left[\left(\mathrm{x}_{i}\right)_{j}-\left(x_{i}\right)_{j \mathrm{REF}}\right]^{2}}{n}},
$$


where $\left(x_{i}\right)_{j},\left(x_{i}\right)_{j \mathrm{REF}}\left(i=1,2,3, x_{1}=x, x_{2}=y, x_{3}=z\right)$ mean the satellite's Cartesian coordinates for epoch $j$ in the inertial reference frame (IRF), in the improved orbit and the reference orbit, respectively, and $n$ represents the total number of epochs of the examined orbital arcs. The value of $\mathrm{RMS}_{\text {OUT }}$ can be interpreted as the average spatial distance between the points of both orbits for the same epoch or as the measure of the fit of the determined orbit to the reference orbit. The reference orbit adopted for $\mathrm{RMS}_{\text {OUT }}$ parameter computation is provided by ESA as a Level 2 GOCE mission product in the previously-mentioned versions: kinematic and reduced-dynamic. The accuracy of this orbit reaches the level of $2 \mathrm{~cm}$ (Bock et al. 2011). An important indicator in this aspect is also the quantity $\mathrm{RMS}_{\mathrm{O}}$, which corresponds to the $\mathrm{RMS}_{\text {OUT }}$ value for the approximate orbit, computed without the use of gravity gradient measurements. The approximate orbit is determined in the numerical integration process using appropriate dynamic models. The initial state vector of this orbit is equal to the initial state vector of the corresponding reference orbit. The quantity $\mathrm{RMS}_{\mathrm{O}}$ is the threshold of orbit improvement effectiveness. The orbit improvement is effective when the obtained $\mathrm{RMS}_{\text {OUT }}$ value is less than the corresponding $\mathrm{RMS}_{\mathrm{O}}$ value.

\section{NUMERICAL TESTS - USING SIMULATED MEASUREMENTS}

In order to check the possibility of GOCE orbit improvement using gravity gradient data, realistic simulations of the gravity gradient observations were first used. These simulations consisted in the computation of the gravity gradient values along the GOCE reference orbit. As already mentioned, this orbit corresponds to the Precise Science Orbit (PSO) of the GOCE satellite, provided as an L2 product by the European Space Agency (ESA 2010). The gravity gradients were determined using the formulas derived by Metris et al. (1999) based on expansion into a spherical harmonic series. Originally computed in the ITRF2005 reference frame, the gravity gradients were then transformed to the IRF reference frame, using the time series of the elements of orientation of the ITRF2005 reference frame with respect to the inertial reference frame (IRF) (ESA 2010). The geopotential model ITGGRACE2010S complete to the degree and order 180 of spherical harmonic coefficients was used for the computations.

The improvement subjects were three 1-day orbital arcs, denoted as variants A, B, C, spanning the following epochs: 6 November 2009, 23:59:45.00 UTC - 7 November 2009, 23:59:45.00 UTC (variant A); 2 December 2009, 23:59:45.00 UTC - 3 December 2009, 23:59:45.00 UTC (variant B); 18 December 2009, 23:59:45.00 UTC - 19 December 2009, 23:59:45.00 UTC (variant C). The corresponding time series of gravity gradients is assigned to each of these variants. The initial state vectors undergoing the improvement process are equal to the initial state vectors of the 
corresponding reference orbit arcs (Table 1). These vectors come from the reduced-dynamic reference orbit (Bock et al. 2011, ESA 2010).

Table 1

Components of the initial state vectors of the GOCE satellite used in the orbit improvement process w.r.t. the J2000.0 inertial reference frame (IRF)

\begin{tabular}{|c|r|r|r|r|r|c|}
\hline No. & \multicolumn{1}{|c|}{$\begin{array}{c}x_{0} \\
{[\mathrm{~km}]}\end{array}$} & \multicolumn{1}{c|}{$\begin{array}{c}y_{0} \\
{[\mathrm{~km}]}\end{array}$} & \multicolumn{1}{c|}{$\begin{array}{c}z_{0} \\
{[\mathrm{~km}]}\end{array}$} & $\begin{array}{c}\dot{x}_{0} \\
{[\mathrm{~km} / \mathrm{s}]}\end{array}$ & $\begin{array}{c}\dot{y}_{0} \\
{[\mathrm{~km} / \mathrm{s}]}\end{array}$ & $\begin{array}{c}\dot{z}_{0} \\
{[\mathrm{~km} / \mathrm{s}]}\end{array}$ \\
\hline $1^{\mathrm{A}}$ & 145.262655 & 880.057268 & -6591.346902 & 5.901115 & -4.956285 & -0.533774 \\
$2^{\mathrm{B}}$ & 6423.724497 & -1652.533840 & -29.552512 & -0.198946 & -0.872715 & 7.705914 \\
$3^{\mathrm{C}}$ & 1039.146802 & 787.067728 & -6509.416262 & 7.641320 & 0.064912 & 1.229579 \\
\hline
\end{tabular}

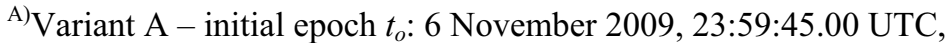

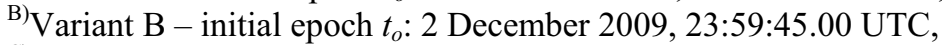

${ }^{\mathrm{C})}$ Variant C - initial epoch $t_{o}: 18$ December 2009, 23:59:45.00 UTC.

\subsection{Computation results}

Using of the initial data presented above in the OCS software made it possible to obtain a series of solutions of orbit determination process. Table 2 presents the accuracies of these solutions obtained using the described simulations of the full gravity gradient tensor measurements. Each of the variants A, B, C associated with the given initial state vector (Table 1) is divided into

Table 2

Accuracy of the GOCE orbit improvement solutions using the simulated observations of the full gravity gradient tensor

\begin{tabular}{|c|c|c|r|r|}
\hline \multirow{2}{*}{ No. } & \multirow{2}{*}{$\begin{array}{c}\text { Orbital arc } \\
\text { length }\end{array}$} & \multicolumn{3}{|c|}{ RMS $_{\mathrm{O}} /$ RMS $_{\text {OUT }}$} \\
\cline { 3 - 5 } & & Variant A & \multicolumn{1}{|c|}{ Variant B } & Variant C \\
\hline 1 & $5.6 \mathrm{~min}$ & $143.734 \mathrm{~m} / 1.3 \mathrm{~cm}$ & $4.2 \mathrm{~cm} / 4.8 \mathrm{~mm}$ & $2.5 \mathrm{~cm} / 3.9 \mathrm{~mm}$ \\
2 & $11.2 \mathrm{~min}$ & $274.514 \mathrm{~m} / 1.2 \mathrm{~cm}$ & $7.1 \mathrm{~cm} / 8.2 \mathrm{~mm}$ & $3.8 \mathrm{~cm} / 4.9 \mathrm{~mm}$ \\
3 & $22.5 \mathrm{~min}$ & $478.906 \mathrm{~m} / 1.5 \mathrm{~cm}$ & $13.5 \mathrm{~cm} / 2.2 \mathrm{~cm}$ & $4.9 \mathrm{~cm} / 1.4 \mathrm{~cm}$ \\
4 & $45.0 \mathrm{~min}$ & $499.384 \mathrm{~m} / 7.7 \mathrm{~cm}$ & $48.9 \mathrm{~cm} / 4.8 \mathrm{~cm}$ & $22.5 \mathrm{~cm} / 7.2 \mathrm{~cm}$ \\
5 & $90.0 \mathrm{~min}$ & $499.314 \mathrm{~m} / 12.5 \mathrm{~cm}$ & $1.357 \mathrm{~m} / 10.7 \mathrm{~cm}$ & $59.7 \mathrm{~cm} / 12.0 \mathrm{~cm}$ \\
6 & $6.0 \mathrm{~h}$ & $498.802 \mathrm{~m} / 37.4 \mathrm{~cm}$ & $5.284 \mathrm{~m} / 34.2 \mathrm{~cm}$ & $2.300 \mathrm{~m} / 27.5 \mathrm{~cm}$ \\
7 & $12.0 \mathrm{~h}$ & $492.079 \mathrm{~m} / 7.054 \mathrm{~m}$ & $10.024 \mathrm{~m} / 56.9 \mathrm{~cm}$ & $3.476 \mathrm{~m} / 56.7 \mathrm{~cm}$ \\
8 & $18.0 \mathrm{~h}$ & $489.077 \mathrm{~m} / 6.926 \mathrm{~m}$ & $13.521 \mathrm{~m} / 1.049 \mathrm{~m}$ & $3.595 \mathrm{~m} / 1.154 \mathrm{~m}$ \\
9 & $24.0 \mathrm{~h}$ & $487.575 \mathrm{~m} / 6.704 \mathrm{~m}$ & $14.813 \mathrm{~m} / 2.759 \mathrm{~m}$ & $3.993 \mathrm{~m} / 2.960 \mathrm{~m}$ \\
\hline
\end{tabular}


nine sub-variants, differing in the length of the improved orbital arc. Therefore, Table 2 contains the characteristics of the accuracy of 27 variants of the solution of the orbit improvement process. A given solution variant can be denoted by providing the arc variant $\mathrm{A}, \mathrm{B}, \mathrm{C}$ and the number corresponding to the given arc length. For the solutions A1 to A9, the simulations of gravity gradient measurements are determined with a sampling interval of $1 \mathrm{~s}$ and the improved orbital arcs are compared with the corresponding arcs of the kinematic reference orbit. The other solution variants, B1-B9 and C1-C9, use simulations of gravity gradient observations with a 10 -s interval. The improved orbital arcs for these variants are compared with the reduceddynamic reference orbit. Comparison of the $\mathrm{RMS}_{\mathrm{O}}$ and $\mathrm{RMS}_{\mathrm{OUT}}$ values in Table 2 indicates effective orbit improvement for all solution variants. The fit of the improved orbit to the reference orbit increased compared with the fit of the approximate orbit from around 1.3 times (variant C9) even to around 320 times (variant A3). The highest orbit improvement effectiveness (value of the $\mathrm{RMS}_{\mathrm{O}} / \mathrm{RMS}_{\text {OUT }}$ ratio) is shown by variants A1-A9, representing the fit of the improved orbit to the kinematic reference orbit. Relatively high $\mathrm{RMS}_{\mathrm{O}}$ values of the fit of the approximate orbit were obtained in this case, which was caused by the adoption of the initial state vector from the reduced-dynamic orbit for the computations. Comparing the $\mathrm{RMS}_{\text {OUT }}$ values, better accuracies can also be observed for the solution variants related to the reduced-dynamic reference orbit than the accuracies of the variants where the kinematic orbit was the reference orbit. For example, variants B7 and C7 related to the reduced-dynamic orbit, showing the $\mathrm{RMS}_{\text {OUT }}$ values of 56.9 and $56.7 \mathrm{~cm}$, respectively, have a much better fit than variant $\mathrm{A} 7$, where the fit to the kinematic reference orbit is at around $7 \mathrm{~m}$. It appears that the better fit of the obtained solutions to the reduced-dynamic orbit may be connected with its higher coherence - a kind of "smoothness", determined by the presence of a strong dynamic aspect in its determination process. Unlike the reduced-dynamic orbit, the kinematic orbit is a strictly geometric solution, covering only the time series of the satellite position vectors. Hence, the kinematic orbit depends more strongly than the reduced-dynamic orbit on the changing quality of GPS observations, which may sometimes lead to abrupt changes in the position vector. Therefore, the kinematic orbit as the reference orbit shows lower consistency with the improved orbit than the reduced-dynamic orbit, which is particularly seen for longer orbital arcs (variants A7-A9 compared with variants B7-B9 and C7-C9).

However, regardless of the type of the orbit used as the reference orbit, the accuracies of the solutions shown in Table 2 decrease with increasing length of the improved orbital arc (except for variants A7-A9, where a slight increase in accuracy can be observed). This effect is caused by different parameterization of the improved orbital arcs (six components of the initial 
state vector) and a different set of used dynamic models compared with the reduced-dynamic reference orbit. Assuming an accuracy of the kinematic orbit and the reduced-dynamic orbit of $2 \mathrm{~cm}$ (Bock et al. 2011), an arc can be indicated among the orbital arcs presented in Table 2 for which the fit of the improved orbit to the reference orbit expressed by the $\mathrm{RMS}_{\text {OUT }}$ values reaches the level of aforementioned accuracy. This arc is the arc with the length of $22.5 \mathrm{~min}$, which is around one-fourth of the satellite period. Moreover, the improvement accuracy for two shorter arcs with the lengths of 5.6 and $11.2 \mathrm{~min}$ is better than in the case of arc with the length of $22.5 \mathrm{~min}$. This also indicates no need to use more extensive parameterization than this, comprising six components of the initial state vector, for orbital arc lengths lower or equal to $22.5 \mathrm{~min}$.

\subsubsection{Sensitivity of orbit determination to spectral content of measure- ments}

Table 3 lists the accuracies of a number of variants of improving the 12-hour orbital arc of the GOCE satellite, in the form of the values of the $\mathrm{RMS}_{\text {OUT }}$ parameter, obtained using the time series of the simulations of the full gravity gradient tensor. These gradients were determined using the geopotential model JYY_GOCE02S (Yi et al. 2013), along the reduced-dynamic reference orbit, with the initial state vector corresponding to variant $\mathrm{C}$ from Table 1 (initial epoch: 18 December 2009, 23:59:45.00 UTC). As a result, eleven sets of gravity gradients were obtained. The gravity gradients of given set have a specified spectral content because they were generated us-

\section{Table 3}

Accuracy of solution variants for orbit determination based on the simulated gravity gradient measurements with different spectral content

\begin{tabular}{|c|c|}
\hline $\begin{array}{c}\text { Degree and order of geopotential } \\
\text { model truncation for the simulation } \\
\text { of the gravity gradient observations }\end{array}$ & $\begin{array}{c}\mathrm{RMS}_{\text {OUT }} \\
{[\mathrm{cm}]}\end{array}$ \\
\hline 1. only $C_{00}$ & 55.7 \\
2. only $C_{00}$ and $C_{20}$ & 55.4 \\
3. $2 \times 2$ & 55.4 \\
4. $5 \times 5$ & 55.4 \\
5. $20 \times 20$ & 55.4 \\
6. $40 \times 40$ & 55.4 \\
7. $70 \times 70$ & 55.4 \\
8. $120 \times 120$ & 55.4 \\
9. $160 \times 160$ & 55.4 \\
10. $200 \times 200$ & 55.4 \\
11. $230 \times 230$ (full model) & 55.4 \\
\hline
\end{tabular}


ing the aforementioned geopotential model truncated at selected degree and order values of spherical harmonic coefficients. Using these sets of gravity gradients in the orbit determination process allowed to obtain eleven solution variants which accuracies are at the same level of $55.4 \mathrm{~cm}$, with simultaneous approximate orbit accuracy of $10.049 \mathrm{~m}$, computed using the full geopotential model JYY_GOCE02S. Variant 1 with the $\mathrm{RMS}_{\text {OUT }}$ value of $55.7 \mathrm{~cm}$ has a slightly lower accuracy than the others. The gradient observations in this variant are determined based on the main Newtonian term of the model JYY_GOCE02S. Starting from variant 2, the accuracy is at the abovementioned constant level. This means clear dependence of orbit improvement accuracy on the effect of the long-wavelength part of the gravity field. As can be seen from Table 3, already the inclusion in the observations of only this long-wavelength part of the gravity field, which is connected with the $C_{00}$ and $C_{20}$ coefficients, suffices to maintain an accuracy of $55.4 \mathrm{~cm}$. Adding the next coefficients in further variants to generate measurements, corresponding to medium- and short-wavelength part of the gravity field does not bring any change in the orbit determination accuracy. In other words, orbit improvement is sensitive to this component of gravity gradient measurements which results from the influence of long-wavelength part of the gravity field. This is consistent with the well-known fact of the dominant effect of this part of the gravity field on the satellite orbit itself.

\subsubsection{Sensitivity of orbit determination to random error modification of observations}

The subject of research was also the improvement of the accuracy for the orbital arc with a length of around 11.2 min depending on the standard deviation of random errors with the normal distribution, which modified the values of simulated gravity gradient observations. To perform this modification, a procedure "gasdev" generating the Gaussian noise was used (Press et al. 1986-1992). The corrected initial state vector was the vector $1^{\mathrm{A}}$ from Table 1 in epoch: 6 November 2009, 23:59:45.00 UTC. Both the approximate arc (computed without improvement) and the improved arcs were compared with the corresponding arc of the reduced-dynamic reference orbit. Hence, the threshold $\mathrm{RMS}_{\mathrm{O}}$ value for the computed orbit was around $4.7 \mathrm{~cm}$. An $\mathrm{RMS}_{\text {OUT }}$ value of the same order for the improved orbit at $5.9 \mathrm{~cm}$ appeared when the simulated observations were modified by random errors with the standard deviation of $10^{-13} \mathrm{~s}^{-2}$, which corresponds to $10^{-4} \mathrm{EU}$ (EU - Eötvös unit, $\left.1 \mathrm{EU}=10^{-9} \mathrm{~s}^{-2}\right)$. For the standard deviation of $10^{-14} \mathrm{~s}^{-2}\left(10^{-5} \mathrm{EU}\right)$, the orbit improvement effectiveness threshold was exceeded because the obtained $\mathrm{RMS}_{\text {OUT }}$ value fell to $7.8 \mathrm{~mm}$. This value is within the accuracy of the reduced-dynamic reference orbit itself, also called the Precise Science Orbit (PSO) (Bock et al. 2011). 


\section{NUMERICAL TESTS - USING REAL MEASUREMENTS}

Taking into account promising results for the simulated gravity gradients, the time series of gravity gradient observations $V_{x x}, V_{y y}, V_{z z}, V_{x y}, V_{x z}, V_{y z}$, from the GOCE mission (Bouman et al. 2011) have been used in the GOCE orbit determination. The gravity gradients used belong to the Level 2 product; i.e., they are determined in a static gravity field. The auxiliary data were the elements of orientation of the GRF and ITRF2005 reference frames with respect to the IRF reference frame. The improvement subject were the same 1day orbital arcs, denoted as variants A, B, C, as in the case of use of simulated gravity gradient observations (Section 4 ). The corresponding time series of real gravity gradients were assigned to each of these variants. The initial state vectors undergoing the improvement process are equal to the initial state vectors of the corresponding reference orbit arcs (Table 1). As already mentioned, these vectors come from the reduced-dynamic reference orbit (Bock et al. 2011, ESA 2010). The values of $\mathrm{RMS}_{\mathrm{O}}$ (the fit of the approximate orbit to the reference orbit) for the described variants of the orbital arcs A, B, and C are: 10.02, 14.81, and $3.99 \mathrm{~m}$, respectively.

Table 4 contains the RMS OUT values for 21 variants of solutions of the improvement process for the three listed 1-day GOCE orbital arcs. The improved orbital arcs were compared with the corresponding arcs of the reduced-dynamic reference orbit. As can be seen in column two, the time series of the diagonal components $V_{x x}, V_{y y}, V_{z z}$ and the time series of individual gravity gradient tensor components were used for orbit determination. These series were transformed from the GRF reference frame to the inertial reference frame (IRF). The sampling interval of the used observations is at $1 \mathrm{~s}$. The obtained results are characterized by $\mathrm{RMS}_{\text {OUT }}$ values at a level of

Table 4

Accuracy of the GOCE orbit improvement solutions using the real gravity gradient measurements

\begin{tabular}{|c|c|r|r|r|}
\hline \multirow{2}{*}{ No. } & \multirow{2}{*}{ Observations } & \multicolumn{3}{|c|}{ RMS $_{\text {OUT }}[\mathrm{km}]$} \\
\cline { 3 - 5 } & & Variant A & Variant B & Variant C \\
\hline 1 & $V_{x x}, V_{y y}, V_{z z}$ & 7732.638 & 272.101 & 142.329 \\
2 & $V_{x x}$ & 636.924 & 1729.157 & 903.993 \\
3 & $V_{y y}$ & 3726.838 & 1412.344 & 128.650 \\
4 & $V_{z z}$ & 700.668 & 374.939 & 227.052 \\
5 & $V_{x y}$ & 867.812 & 360.509 & 644.726 \\
6 & $V_{x z}$ & 391.541 & 1246.821 & 339.446 \\
7 & $V_{y z}$ & 12856.773 & $1.014 \times 10^{9}$ & 9135.146 \\
\hline
\end{tabular}


of hundreds, and even thousands of kilometers which, compared with the corresponding $\mathrm{RMS}_{\mathrm{O}}$ values given in meters, clearly rules out the possibility of effectively using the real gravity gradient measurements from the GOCE mission for orbit improvement. This is caused by the relatively low accuracy of the measured gravity gradients in the time domain, as indicated by an analysis of a time series including 2584174 measurement epochs with a 1-s sampling interval, contained within the time span from epoch 1 November 2009, 00:49:15.937778 UTC to epoch 30 November 2009, 22:38:47.175033 UTC. The absolute value of the average sum of the diagonal components is around $10^{-6} \mathrm{~s}^{-2}$. For comparison, the absolute value of the sum of these components determined analytically is in the order of $10^{-38} \mathrm{~s}^{-2}$ while for errorfree measurement values it is 0 .

Ignoring the orbital aspect in this case, the results presented in Table 4 can be useful to some degree in comparing the quality of the used time series of observations. Comparison of the $\mathrm{RMS}_{\text {Out }}$ values for individual variants of improved orbital arcs would indicate the best quality of measurements for arc C (the epochs: 18 December 2009, 23:59:45.00 UTC - 19 December 2009, 23:59:45.00 UTC) and/or maybe the better quality of orientation elements used to the transformation of observations from the GRF to IRF reference frame. Among the gravity gradients, the best result was obtained after the application of $V_{y y}$ (RMS Out is $128.65 \mathrm{~km}$ for $\left.\operatorname{arc} \mathrm{C}\right)$. The solutions obtained using the diagonal components $V_{x x}, V_{y y}, V_{z z}\left(\mathrm{RMS}_{\text {OUT }}-142.329 \mathrm{~km}\right)$, the component $V_{z z}\left(\mathrm{RMS}_{\text {OUT }}-227.052 \mathrm{~km}\right)$ and the component $V_{x z}\left(\mathrm{RMS}_{\text {OUT }}\right.$ - $339.446 \mathrm{~km}$ ) also stand out. The relatively small $\mathrm{RMS}_{\text {OUt }}$ for the solution C1 with the components $V_{x x}, V_{y y}, V_{z z}$ can be connected with their nominally higher accuracy and better conditioning of the orbit determination process. The solutions obtained based on the gravity gradient $V_{y z}$ show by far the lowest accuracy. This is approximately consistent with the predicted division of the measured gravity gradient tensor components into the nominally more accurate $V_{x x}, V_{y y}, V_{z z}, V_{x z}$ and less accurate $V_{x y}, V_{y z}$ (Bouman et al. 2011). This division results from a design limitation of the gradiometer, in which the precision of measurement along one of the three accelerometer axes is lower by two orders of magnitude than the precision of measurement along the two others (ESA 2008). In this context, the distinguishing $\mathrm{RMS}_{\mathrm{OUT}}$ value of $360.509 \mathrm{~km}$ for the solution using the component $V_{x y}$ in the arc B improvement is also a certain surprise. This has not been yet well understood. On the other hand, the remaining two solutions, A5 and C5, based on the component $V_{x y}$ have the $\mathrm{RMS}_{\text {OUT }}$ values of 867.812 and $644.726 \mathrm{~km}$, respectively, which generally indicates a smaller accuracy than the solutions based on the nominally more accurate gravity gradients. 


\section{SUMMARY AND CONCLUSIONS}

The time series of simulated and real gravity gradient observations were used in the GOCE satellite orbit improvement process, applying the classical least squares method. In case of using of simulated gravity gradient observations, the GOCE orbit improvement is effective in all tested cases. The accuracy of the improved orbit variants ranges from the level of millimeters (for the arc lengths of 5.6 and $11.2 \mathrm{~min}$ ), through the level of centimeters (for the arc lengths of 22.5 and $45.0 \mathrm{~min}$ ), decimeters (the arc lengths: $90.0 \mathrm{~min}$, $6.0 \mathrm{~h}, 12.0 \mathrm{~h}$ ), up to the level of meters (the arc lengths of 18.0 and $24.0 \mathrm{~h}$ ). At the same time, the orbital arc with the length of $22.5 \mathrm{~min}$ is the longest among those tested for which improvement accuracy, from 1.4 to $2.2 \mathrm{~cm}$ depending on the variant, is within the accuracy of the used reference orbit (PSO orbit of the GOCE satellite). Hence, for the orbital arc of this length it is sufficient to correct only the initial state vector, without the need to introduce additional dynamic parameters, to maintain the reference orbit accuracy. The improved orbital arcs with lengths from $45.0 \mathrm{~min}$ to $24.0 \mathrm{~h}$ can be treated as more-or-less accurate approximate orbits.

Further research for the 12-hour arc showed practically complete dependence of the orbit improvement accuracy on this component of gravity gradient measurements, which is generated by the long-wavelength part of the gravity field; it is sufficient to include only the $C_{00}$ and $C_{20}$ coefficients in the observation model.

Disturbing the sets of simulated measurements by random errors indicates that the value of the standard deviation of the measurements in the order of $10^{-5} \mathrm{EU}$ is necessary to achieve an accuracy of the improved orbital arc with the length of $11.2 \mathrm{~min}$ under $1 \mathrm{~cm}$, i.e., close to the reference orbit accuracy.

When real observations were used, the obtained solutions had an accuracy of hundreds and even thousands of kilometers. This clearly excludes the possibility of using the real gradient measurements from the GOCE mission for satellite orbit improvement. This is caused by the low accuracy of these observations in the time domain, which is connected with the low accuracy in the low frequency part of spectrum. An additional factor lowering the orbit estimation accuracy is the need to transform gravity gradients from the gradiometer reference frame to the inertial reference frame, where the orbit improvement process is performed.

The obtained results show effective improvement of different GOCE orbital arcs, on condition that a time series of measurements of the full gravity gradient tensor with appropriate accuracy in the long-term frequency range is applied. It is also best for the tensor to be measured directly in the orbit improvement reference frame, i.e., in the inertial reference frame. Of course, 
the application of gravity gradients in satellite orbit determination should be treated as an additional possibility for validating the orbit determined using the basic data such as GPS observations. The use of gravity gradient observations for improvement of short orbital arcs, for example, can also be considered, especially where GPS measurements show lower accuracy for different reasons.

\section{References}

Bobojć, A., and A. Drożyner (2003), Satellite orbit determination using satellite gravity gradiometry observations in GOCE mission perspective, Adv. Geosci. 1, 109-112, DOI: 10.5194/adgeo-1-109-2003.

Bock, H., A. Jäggi, D. Švehla, G. Beutler, U. Hugentobler, and P. Visser (2007), Precise orbit determination for the GOCE satellite using GPS, $A d v$. Space Res. 39, 10, 1638-1647, DOI: 10.1016/j.asr.2007.02.053.

Bock, H., A. Jäggi, U. Meyer, P. Visser, J. van den Ijssel, T. van Helleputte, M. Heinze, and U. Hugentobler (2011), GPS-derived orbits for the GOCE satellite, J. Geodesy 85, 11, 807-818, DOI: 10.1007/s00190-011-0484-9.

Bouman, J., S. Fiorot, M. Fuchs, T. Gruber, E. Schrama, Ch. Tscherning, M. Veicherts, and P. Visser (2011), GOCE gravitational gradients along the orbit, J. Geodesy 85, 11, 791-805, DOI: 10.1007/s00190-011-0464-0.

Drożyner, A. (1995), Determination of orbits with Toruń Orbit Processor system, Adv. Space Res. 16, 12, 93-95, DOI: 10.1016/0273-1177(95)98788-P.

ESA (1999), Gravity field and steady-state ocean circulation mission, Report for mission selection of the four candidate Earth Explorer missions, ESA SP1233(1), European Space Agency, Noordwijk.

ESA (2008), GOCE L1B products user handbook, SERCO/DATAMAT Consortium, ESA Tech. Note GOCE-GSEG-EOPG-TN-06-0137, European Space Agency, Noordwijk.

ESA (2010), GOCE Level 2 Product Data Handbook, European GOCE Gravity Consortium; ESA Tech. Note GO-MA-HPF-GS-0110, European Space Agency, Noordwijk.

Eshagh, M. (2014), From tensor to vector of gravitation, Artif. Sat. 49, 2, 63-80, DOI: $10.2478 /$ arsa-2014-0006.

Eshagh, M., and M. Najafi-Alamdari (2007), Perturbations in orbital elements of a low Earth orbiting satellite, J. Earth Space Phys. 33, 1, 1-12.

Heiskanen, W., and H. Moritz (1967), Physical Geodesy, WH Freeman and Co., San Francisco.

Johannessen, J.A., G. Balmino, C. le Provost, R. Rummel, R. Sabadini,H. Sünkel, C.C. Tscherning, P. Visser, P. Woodworth, C.W. Hughes, P. Legrand, 
N. Sneeuw, F. Perosanz, M. Aguirre-Martinez, H. Rebhan, and M.R. Drinkwater (2003), The european gravity field and steady-state ocean circulation explorer satellite mission: its impact on geophysics, Surv. Geophys. 24, 4, 339-386, DOI: 10.1023/B:GEOP.0000004264.04667.5e.

Mayer-Gürr, T., A. Eicker, and J. Schall (2010), Regional high resolution geoid and mean sea surface topography determination by a combination of GOCE, GRACE and altimetry data. In: ESA Living Planet Symposium, 28 June - 2 July 2010, Bergen, Norway.

Métris, G., J. Xu, and I. Wytrzyszczak (1999), Derivatives of the gravity potential with respect to rectangular coordinates, Celest. Mech. Dyn. Astr. 71, 2, 137-151, DOI: 10.1023/A:1008361202235.

Palacios, M., A. Abad, and A. Elipe (1992), An efficient numerical method for orbit computations. In: Proc. AAS/AIAA Astrodynamics Conference, 19-22 August 1991, Durango, USA, Part 1 (A92-43251 18-13), Univelt Inc., San Diego, 265-274.

Press, W.H., S.A. Teukolsky, W.T. Vetterling, and B.P. Flannery (1986-1992), Numerical Recipes in FORTRAN 77: The Art of Scientific Computing, Cambridge University Press, New York.

Rebhan, H., M. Aguirre, and J. Johannessen (2000), The Gravity field and steadystate ocean circulation explorer mission - GOCE, ESA Earth Observ. Quart. 66, 6-11.

Rummel, R., and O.L. Colombo (1985), Gravity field determination from satellite gradiometry, Bull. Géod. 59, 3, 233-246, DOI: 10.1007/BF02520329.

Rummel, R.F., D. Muzi, M.R. Drinkwater, R. Floberghagen, and M. Fehringer (2009), GOCE: mission overview and early results. In: American Geophysical Union, Fall Meeting, 14-18 December 2009, San Francisco.

Rummel, R., W. Yi, and C. Stummer (2011), GOCE gravitational gradiometry, J. Geodesy 85, 11, 777-790, DOI: 10.1007/s00190-011-0500-0.

Yi, W., R. Rummel, and T. Gruber (2013), Gravity field contribution analysis of GOCE gravitational gradient components, Studia Geophys. Geod. 57, 2, 174-202, DOI: 10.1007/s11200-011-1178-8.

Received 7 July 2014 Received in revised form 13 February 2015 Accepted 10 March 2015 